\title{
Center Symmetry and Abelian Projection at Finite Temperature
}

\author{
Michael C. Ogilvie ${ }^{\mathrm{a} *}$ \\ ${ }^{a}$ Washington University, St. Louis, MO, USA
}

\begin{abstract}
At finite temperature, there is an apparent conflict between Abelian projection and critical universality. For example, should the deconfinement transition of an $S U(2)$ gauge theory projected to $U(1)$ lie in the $Z(2)$ universality class of the parent $S U(2)$ theory or in the $U(1)$ universality class? I prove that the projected theory lies in the universality class of the parent gauge theory. The mechanism is shown to be non-local terms in the projected effective action involving Polyakov loops. I connect this to the recent work by Dunne et al. on the deconfinement transition in the $2+1$ dimensional Georgi-Glashow model.
\end{abstract}

There is an apparent conflict between Abelian projection and critical universality. In its simplest form, critical universality states that the universality class of a phase transition depends only on the dimensionality of the system and the symmetry group of the order parameter. Universality thus tells us that the nature of the deconfinement transition for a pure gauge theory with gauge group $\mathrm{G}$ depends only on the dimensionality of space and the center of the gauge group $\mathrm{C}(\mathrm{G})[1]$.

For example, $S U(2)$ gauge theories are in the $Z(2)$ universality class of the Ising model, while $S U(3)$ gauge theories are in Potts model, or $Z(3)$, universality class. In Abelian projection, the gauge group is reduced. This reduction can changes the center of the gauge group, and thus naively may change the universality class. A common example would be the reduction of $S U(2)$ to $U(1)$. The center of $S U(2)$ is $Z(2)$, but the center of $U(1)$ is $U(1)$ itself. Thus the deconfinement transition of the projected theory appears to be in a different universality class from the original underlying gauge theory. However, this naive expectation is wrong: lattice simulations show that the critical exponents for $S U(2)$ projected to $U(1)$ are identical to the critical exponents of the underlying $S U(2)$ gauge theory [2]. The explanation for this behavior is given below.

In lattice gauge theory, Abelian projection has

\footnotetext{
*We gratefully acknowledge the support of the U.S. Dept.
} of Energy under DOE DE-FG02-91ER40628. a natural algorithmic formulation. For analytical purposes, I will use the formalism developed in [3], which uses gauge fields $u_{\mu}(x) \in G$, site-based gauge-fixing fields $g(x) \in G$, andAbelian gauge fields $h_{\mu}(x) \in H \subset G$. Three different actions play a role: the gauge action

$S_{g}=\frac{\beta}{2 N} \sum_{p l a q} \operatorname{Tr}\left(u_{p}+u_{p}^{+}\right)$,

the gauge-fixing action, which for $S U(2)$ is given by

$S_{g f}=\lambda \sum_{\text {links }} \operatorname{Tr}\left[u_{l} \sigma_{3} u_{l}^{+} \sigma_{3}\right]$,

and the projection action

$S_{\text {proj }}[u, h]=\sum_{\text {links }}\left[\frac{p}{2 N} \operatorname{Tr}\left(u_{l}^{+} h_{l}+u_{l}^{+} g_{l}\right)\right]$

As $p, \lambda \rightarrow \infty$, the usual lattice projection algorithm is formally obtained. The variables $g$ and $h$ are quenched: $u$ 's are generated by $S_{g}$, then $g$ 's are generated by $S_{g f}$. Finally, $h$ 's are generated from the gauge-fixed $u$ 's. Expectation values are given by

$$
\begin{aligned}
\langle O\rangle= & \frac{1}{Z_{g}} \int[d u] e^{S_{g}[u]} \frac{1}{Z_{g f}[u]} \int[d g] e^{S_{g f}[\widetilde{u}]} \\
& \cdot \frac{1}{Z_{\text {proj }}[\widetilde{u}]} \int[d h] e^{S_{\text {proj }}[\widetilde{u}, h]} O .
\end{aligned}
$$

Relations between the Green functions of the projected theory and the Green functions of 
the underlying non-Abelian gauge theory can be found using character expansions. The projection weight can be expanded as

$$
\begin{aligned}
\exp & {\left[\frac{p}{2 N} \operatorname{Tr}\left(u^{+} h+u^{+} g\right)\right] } \\
& =\sum_{\alpha} d_{\alpha} c_{\alpha}(p) \chi_{\alpha}\left(h^{+} u\right)
\end{aligned}
$$

where $\chi_{\alpha}$ is a group character, $d_{\alpha}$ is the dimensionality of the representation, and $c_{a}(p)$ is a positive, p-dependent coefficient. I use $\widetilde{\chi}^{\beta}(h)$ to denote the corresponding character of the projection subgroup. Note that $\chi^{\alpha}(h)$ is in general a reducible representation of $\mathrm{H}$. For example, the $\mathrm{j}=1$ representation of $\mathrm{SU}(2)$ is the sum of three irreducible representations of $\mathrm{U}(1)$, given by $\mathrm{m}=+1,0,-1$. To leading order in the character expansion for projection, and to order $\lambda^{0}$ for gauge-fixing, we have

$$
\begin{aligned}
& \left\langle\widetilde{\chi}^{\beta}\left(h_{1} . . h_{n}\right)\right\rangle \\
& =\sum_{\alpha}\left(\frac{c_{\alpha}(p)}{c_{0}(p)}\right)^{n} \int_{H}(d h) \widetilde{\chi}^{\beta}(h) \chi^{\alpha}\left(h^{+}\right) \\
& \quad \cdot\left\langle\chi^{\alpha}\left(g_{1} . . g_{n}\right)\right\rangle
\end{aligned}
$$

for any closed Wilson loop, where $n$ is the length of the loop. For a Polyakov loop $u_{P}(\vec{x})$, we have

$$
\left\langle\operatorname{Tr} h_{P}(\vec{x})\right\rangle \simeq\left(\frac{c_{F}(p)}{c_{0}(p)}\right)^{n_{t}}\left\langle\operatorname{Tr} u_{P}(\vec{x})\right\rangle
$$

Just above a second-order critical point, we have

$$
\left\langle\operatorname{Tr} u_{P}(\vec{x})\right\rangle \sim\left(T-T_{c}\right)^{\beta}
$$

so we must have the same critical index $\beta$ in the projected theory as in the original. Similar results hold for higher correlation functions and thus for all critical indices. We conclude that the critical behavior of the projected theory must be that of the original gauge theory.

We can understand this result on the basis of center symmetry. A center symmetry transformation on the original gauge theory acts on all the timelike links on a given time slice as

$u_{0}(\vec{x}, t) \rightarrow z u_{0}(\vec{x}, t)$

where $z$ is an element of the center $\mathrm{C}(\mathrm{G})$ of $\mathrm{G}$. $S_{g}[u]$ is invariant under this transformation but the Polyakov loop $u_{P}(\vec{x})$ is not. The deconfinement transition is the spontaneous breakdown of center symmetry at high temperature. REF

We can define an effective action $S_{\text {eff }}[h]$ for the projected theory as

$$
\begin{aligned}
e^{S_{e f f}[h]}= & \frac{1}{Z_{g}} \int[d u] e^{S_{g}[u]+S_{g f}[u]+S_{p r o j}[u, h]} \\
& \cdot \frac{1}{Z_{g f}[u]} \frac{1}{Z_{\text {proj }}[u]}
\end{aligned}
$$

Now suppose that $\mathrm{w}$ is an element of $\mathrm{C}(\mathrm{H})$. Then consider the transformation $h_{0}(\vec{x}, t) \rightarrow$ $w h_{0}(\vec{x}, t)$ on a single time slice. It is easy to see that the replacement $S_{\text {eff }}[h] \neq S_{\text {eff }}[w h]$ unless $S_{g}[w u]=S_{g}[u]$. Thus $S_{\text {eff }}$ is only invariant under $C(G)$, not $C(H)$.

The effective action can be constructed order by order in a strong-coupling expansion around $\beta, \lambda, p=0$. To leading order for $\mathrm{SU}(\mathrm{N})$ we have

$$
S_{\text {eff }}[h]=\frac{\beta p^{4}}{32 N^{9}} \sum_{\text {plaq }} \operatorname{Tr}\left(h_{\text {plaq }}+h_{\text {plaq }}^{+}\right)
$$

This effective action is invariant under gauge transformations in $H$ and invariant under the global symmetry $h \rightarrow w h$. Note that contributions from Wilson loops of area $A$ are suppressed by a factor of $\beta^{A}$, but such terms are also invariant under $h \rightarrow w h$.

Polyakov loops break the invariance of $S_{\text {eff }}[h]$ under $h \rightarrow w h$. The lowest such contribution comes from integration over $N_{t}$ temporal plaquettes, aligned to form a belt of plaquettes around the lattice in the temporal direction. Integration over the $u$ field leads to an interaction of the form

$$
\begin{aligned}
& \left(\frac{\beta}{2 N^{2}}\right)^{N_{t}}\left(\frac{p}{2 N^{2}}\right)^{2 N_{t}} \\
& \cdot\left[\operatorname{Tr}\left(h_{P}(\vec{x})\right) \operatorname{Tr}\left(h_{P}^{+}(\vec{x}+\widehat{i})\right)+h . c .\right]
\end{aligned}
$$

where $h_{P}(\vec{x})$ is a Polyakov loop formed from the $h$ fields:

$h_{P}(\vec{x})=\prod_{t} h_{0}(\vec{x}, t)$.

This Polyakov loop term is not invariant under the change of variables $h_{P} \rightarrow w h_{P}$ unless $w$ is in $C(G)$. 
Consider the case of $S U(2)$ projected to $U(1)$. If we write

$h_{P}(\vec{x})=\left(\begin{array}{ll}e^{i \theta(\vec{x})} & 0 \\ 0 & e^{-i \theta(\vec{x})}\end{array}\right)$,

the interaction between Polyakov loops has the form

$\cos \theta(\vec{x}) \cos \theta(\vec{x}+\widehat{i})$.

The transformation $h_{P} \rightarrow w h_{P}$ induces a change $\theta \rightarrow \theta+\alpha$ which is a symmetry of the action only when $\alpha=0$ or $\alpha=\pi$, i.e., when $w \in Z(2)$. A true $U(1)$ gauge theory, in comparison, could only have effective interactions like

$2 \cos [\theta(\vec{x})-\theta(\vec{x}+\widehat{i})]$

which is invariant for arbitrary $\alpha$. We thus see how $S_{\text {eff }}[h]$ includes Polyakov loop terms, nonlocal in Euclidean time, which force the global center symmetry to be $C(G)$ rather $C(H)$.

The need to include specific, non-local terms associated with Polyakov loops to effective actions is not limited to the case of Abelian projection. Similar behavior has recently been demonstrated by Dunne et al. [1] in the finitetemperature Georgi-Glashow model in $2+1$ dimensions. Adjoint representation scalars break an $S U(2)$ gauge symmetry to $U(1)$. The lowenergy behavior of the model is that of a $U(1)$ theory, and thus the question of $Z(2)$ versus $U(1)$ center symmetry arises here as well.

This model has a semiclassical regime where confinement can be demonstrated as a consequence of instanton effects. Agasian and Zarembo [5] have shown that at finite temperature, these instantons are seen by Polyakov loops as vortices, of the type that drive Kosterlitz-Thouless phase transitions in 2-dimensional systems. A detailed renormalization group analysis appeared to show that the deconfining phase transition in the $d=2+1$ Georgi-Glashow model was in the $U(1)$ universality class.

However, Dunne et al. have demonstrated that Polyakov loop effects associated with heavy $W^{ \pm}$ gauge bosons must be explicitly included in any discussion of critical behavior. The effect on the finite-temperature, $d=2+1$ Georgi-Glashow model is precisely parallel to adding a symmetrybreaking term to a $U(1)$ spin model, reducing the global symmetry from $U(1)$ to $Z(2)$. It is possible to show explicitly that the critical behavior of the model lies in the $Z(2)$ universality class of the two-dimensional Ising model after all.

Abelian projection does not enlarge center symmetry. As a consequence, projection does not violate universality: the projected theory is in the same universality class as the original, underlying gauge theory. Any effective action for a projected theory must include non-local terms at non-zero temperatures to give the correct critical behavior at deconfinement. Comparison of projection at finite temperature with the Georgi-Glashow model at finite temperature shows the similarity of the issue in the two models, and the similar resolution. This suggests that we may have identified all the terms of the projected effective action which can be relevant in the renormalization group sense. If true, this is an important step in understanding how Abelian projection reproduces the behavior of the underlying gauge theory.

\section{REFERENCES}

1. L. G. Yaffe and B. Svetitsky, Phys. Rev. D 26 (1982) 963.

2. S. Ejiri, S. i. Kitahara, T. Suzuki and K. Yasuta, Phys. Lett. B 400 (1997) 163.

3. M. C. Ogilvie, Phys. Rev. D 59 (1999) 074505 .

4. G. V. Dunne, I. I. Kogan, A. Kovner and B. Tekin, JHEP 0101 (2001) 032.

5. N. O. Agasian and K. Zarembo, Phys. Rev. D 57 (1998) 2475. 